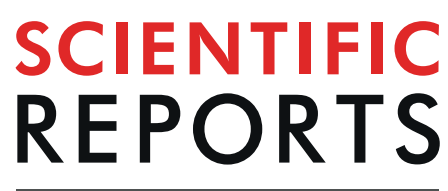

natureresearch

\title{
A xylan glucuronosyltransferase gene exhibits pleiotropic effects on cellular composition and leaf development in rice
}

\author{
Dawei Gao ${ }^{1}$, Wenqiang Sun ${ }^{1}$, Dianwen Wang ${ }^{1}$, Hualin Dong ${ }^{1}$, Ran Zhang ${ }^{2} \&$ Sibin $\mathrm{Yu}^{1 *}$
}

Leaf chlorophyll content is an important physiological indicator of plant growth, metabolism and nutritional status, and it is highly correlated with leaf nitrogen content and photosynthesis. In this study, we report the cloning and identification of a xylan glucuronosyltransferase gene (OsGUX1) that affects relative chlorophyll content in rice leaf. Using a set of chromosomal segment substitution lines derived from a cross of wild rice accession ACC10 and indica variety Zhenshan 97 (ZS97), we identified numerous quantitative trait loci for relative chlorophyll content. One major locus of them for relative chlorophyll content was mapped to a 10.3-kb region that contains OsGUX1. The allele OsGUX1 ${ }^{A C}$ from ACC10 significantly decreases nitrogen content and chlorophyll content of leaf compared with OsGUX1 ${ }^{25}$ from ZS97. The overexpression of OsGUX1 reduced chlorophyll content, and the suppression of this gene increased chlorophyll content of rice leaf. OsGUX1 is located in Golgi apparatus, and highly expressed in seedling leaf and the tissues in which primary cell wall synthesis occurring. Our experimental data indicate that $O s G U X 1$ is responsible for addition of glucuronic acid residues onto xylan and participates in accumulation of cellulose and hemicellulose in the cell wall deposition, thus thickening the primary cell wall of mesophyll cells, which might lead to reduced chlorophyll content in rice leaf. These findings provide insights into the association of cell wall components with leaf nitrogen content in rice.

Rice (Oryza sativa. L) providing a staple food for more than half of the population in the world, is a monocot model species for genetics, biology and functional genomics studies. Leaf photosynthesis has great potential for the improvement of rice yield, which will significantly contribute to addressing food demand challenge. The chloroplast of green tissue or leaf, as the most important supporter of carbon fixation and energy transformation, plays important roles in photosynthesis ${ }^{1-3}$. Both genetic and environmental factors have effect on the biochemical composition in chloroplast, thus affecting photosynthetic rate . $^{4}$

Previous studies have indicated that leaf color is a sensitive indicator of crop growth, metabolism and nutritional status, and it is closely related to the content of photosynthetic pigment, and positively correlated with leaf nitrogen $(\mathrm{N})$ content $^{5-7}$. Understanding the genetic and physiological bases of leaf nitrogen status is essential for efficient crop production and nitrogen management in intensive rice cropping systems. Leaf greenness is determined by specific properties, such as leaf chlorophyll content and chloroplast development, and leaf morphological characteristics (leaf thickness, surface structure and wall components). To study the genetic and molecular basis of leaf color, more than one hundred of rice mutants associated with leaf colors and chlorophyll content have been identified (http://archive.gramene.org/db/genes/). Several genes have been implicated in the chlorophyll biosynthesis, degradation, and regulation of chloroplast development through analyses of chlorophyll-deficiency mutations, which are typically reflected in leaf color $^{8-11}$. For example, SGR (Stay Green Rice) gene encodes an ancient protein containing a putative chloroplast transit peptide. The $s g r$ mutant was characterized by chlorophyll retention with stable chlorophyll-protein complexes and thylakoid membrane structures. Overexpression of SGR reduced the number of thylakoid lamellae in the chloroplasts and the chlorophyll content in growing leaves ${ }^{12}$. However, there have been few reports on cloning of the gene influencing leaf color or leaf $\mathrm{N}$ content in natural

${ }^{1}$ National Key Laboratory of Crop Genetic Improvement, College of Plant Science and Technology, Huazhong Agricultural University, Wuhan, 430070, China. ${ }^{2}$ Biomass \& Bioenergy Research Centre, Huazhong Agricultural University, Wuhan, 430070, China. *email: ysb@mail.hzau.edu.cn 
variation populations in rice. Despite a few studies on the effect of carbohydrate metabolism on chloroplast development and plant development ${ }^{13-16}$, it is still largely lacking of the genetic connection of the leaf color and the cellular components in leaf primary cell walls. The knowledge of the genes related to cellular components that influence chlorophyll content and leaf structure is limited.

Rice is a grass where xylan is the major non-cellulosic component of the leaf primary cell walls. Many glucuronyltransferase genes of Glycosyltransferase Family 8 (GT8) are reported responsible for sugar substitutions on the xylan of the primary cell walls. GT8 members are involved in several unique types of glycoconjugate and glycan biosynthetic processes and include the galacturonosyltransferase (GAUT) and GAUT-Like clade, galactinol synthase (GolS), inositol phosphorylceramide glucuronosyltransferase1 (IPUT1), and glucuronic acid substitution of xylan (GUX) protein clades with distinct functions in plants ${ }^{17,18}$. Particularly, Arabidopsis GUX1, GUX2 and GUX3 make different distinct patterns of glucuronic acid (GlcA) and/or methylglucuronic acid (MeGlcA) substitutions ${ }^{19,20}$. GUX1 and GUX2, both required for substitution of the xylan backbone with [Me]GlcA, are related to secondary wall biosynthesis. The mutants gux 1 and $g u x 2$, lose the xylan glucuronyltransferase activity, affecting the addition of GlcA or MeGlcA to xylan ${ }^{17}$. Such an addition influences normal secondary wall deposition and plant development. GUX3 is involved in addition of the GlcA decorations on xylan of the primary cell wall in Arabidopsis ${ }^{20}$. Further, the GUX1/2/3 proteins exhibit capability of transferring GlcA residues from the UDP-GlcA donor onto xylooligomer acceptors in tobacco BY2 cells ${ }^{21}$. The gux1/2/3 triple mutant has been reported to cause a complete loss of GlcA and MeGlcA side chains on xylan. However, dissimilar phenotype in plant growth was observed for the $g u \times 1 / 2 / 3$ triple mutant in different studies ${ }^{20,21}$. One study showed that the triple mutant reduced secondary wall thickening, collapsed vessel morphology and inhibited plant growth ${ }^{21}$. Another thorough study revealed that the absence of GlcA xylan in cell wall had no impact on plant growth in the gux 1/2/3 triple mutant ${ }^{20}$. Further investigation of this discrepancy is required. Therefore, it is very interested in whether the rice homologous genes of GUXs associated with cellular components affect plant growth in rice.

In the present study, a set of chromosomal segment substitution lines (CSSL) derived from a wild rice accession (designated as ACC10) and an indica cv. Zhenshan 97B (ZS97) was used for detecting quantitative trait loci (QTL) for relative chlorophyll content and nitrogen content in rice leaf. Using a CSSL-derived $\mathrm{F}_{2}$ population, a major QTL for leaf chlorophyll content measured by Soil-Plant Analysis Development (SPAD) value was finely mapped to a 10.3-kb region. Transgenic experiments revealed that $O s G U X 1$ affects primary cell wall components corresponding to the QTL for relative chlorophyll content.

\section{Results}

Fine mapping of $q N C 1.2$ for leaf nitrogen content in CSSLs. As a first step to identify the key genes underlying QTLs for leaf color or nitrogen content, a set of chromosome segment substitution lines, each of which carries a single or few chromosome segments of a wild rice accession (ACC10) in the ZS97 genetic background were developed. To determine the genotype precisely, the CSSLs were re-analyzed using a SNP chip RICE6K (Illumina) containing 5,102 SNPs markers with an average density of 12 SNPs per $\mathrm{Mb}^{22}$. The results display that each line contained one or a few introduced donor segments at a particular chromosomal region against the uniform genetic background of ZS97, and all the substitution segments together covered the entire ACC10 genome, except one gap on chromosome 7 physical region $(22.34-24.39 \mathrm{Mb})$. Genotyping of the CSSL population composed of 111 lines was defined as a total of 379 bins (B1 to B379) across the whole genome (Fig. S2). The physical length of the bins ranged from $19.7 \mathrm{~kb}$ to $8.5 \mathrm{Mb}$ with a median size of $0.69 \mathrm{Mb}$.

Two parental lines (ZS97 and ACC10) differed markedly in relative chlorophyll content and nitrogen content in flag leaf which were determined by SPAD-value (Table S1). ZS97 had higher relative chlorophyll content, which was significantly different from ACC10. The CSSL population varied widely in relative chlorophyll content. Most lines had phenotypic values similar to that of ZS97, whereas several lines exhibited significantly differences in relative chlorophyll content, compared with ZS97. Four putative QTLs for relative chlorophyll content were identified in QTL analysis of the population with 379 bin markers (Table S2). Of these 4 QTLs, a major locus $q N C 1.2$ located on the long arm of chromosome 1 exhibited the largest effect, explaining $26.9 \%$ of the phenotypic variation in the population.

To validate the effect of $q N C 1.2$ on relative chlorophyll content or nitrogen content, we constructed a CSSL-derived $\mathrm{F}_{2}$ segregating population at the target QTL and genotyped the population by using 16 polymorphic SSR markers within the target region. QTL analysis of a small CSSL-derived $\mathrm{F}_{2}$ population consisting of 190 individuals indicated that qNC1.2 for relative chlorophyll content in flag leaf was flanked by RM11847-RM431, explaining $37.4 \%$ of the phenotypic variation (Fig. 1A). Three genotypes at RM11943 tightly linked with qNC1.2 displayed significant differences in SPAD-value (Fig. 1B). The ACC10 alleles decreased relative chlorophyll content in a partial dominant way. Ninety-five recombinants within RM11847-RM431 were genotyped with additional ten SSR markers. From them, 20 representative recombinants with the wild rice introduced fragments overlapping the QTL region were selected for progeny testing. The progeny testing delimited the QTL into a 188.4-kb region between RM6333 and RM11962 (Fig. 1C).

To finely map $q N C 1.2$, we further screened a total of 1200 individuals from the $\mathrm{F}_{2}$ population using the markers RM6333 and RM431 (Fig. 1C). Seven new recombinants were obtained from RM6333-RM431 region by using ten additional SNP or InDel markers. Their homozygous lines were developed and used for the phenotypic investigation. During the process, two near-isogenic lines ( $\mathrm{NIL}^{\mathrm{ZS}}$ and NIL ${ }^{\mathrm{AC}}$ ) containing the contrasting alleles (ZS97 and ACC10) within an approximately 200-kb region of $q N C 1.2$ were developed. The analyses of these homozygous recombinant lines delimited $q$ NC1.2 to a 10.3-kb region between markers SPRD19 and RM11962 (Fig. 1D). This small region contained two predicted genes (LOC_Os01g65770 and LOC_Os01g65780) in the genome database (http://rice.plantbiology.msu.edu/cgi-bin/gbrowse). The former (LOC_Os01g65770) encoded a putative express protein and it was hardly expressed in any tissues, while the latter (LOC_Os01g65780) encoded a putative plant glycosyltransferase and was constitutively expressed at various tissues (http://crep.ncpgr.cn/). 


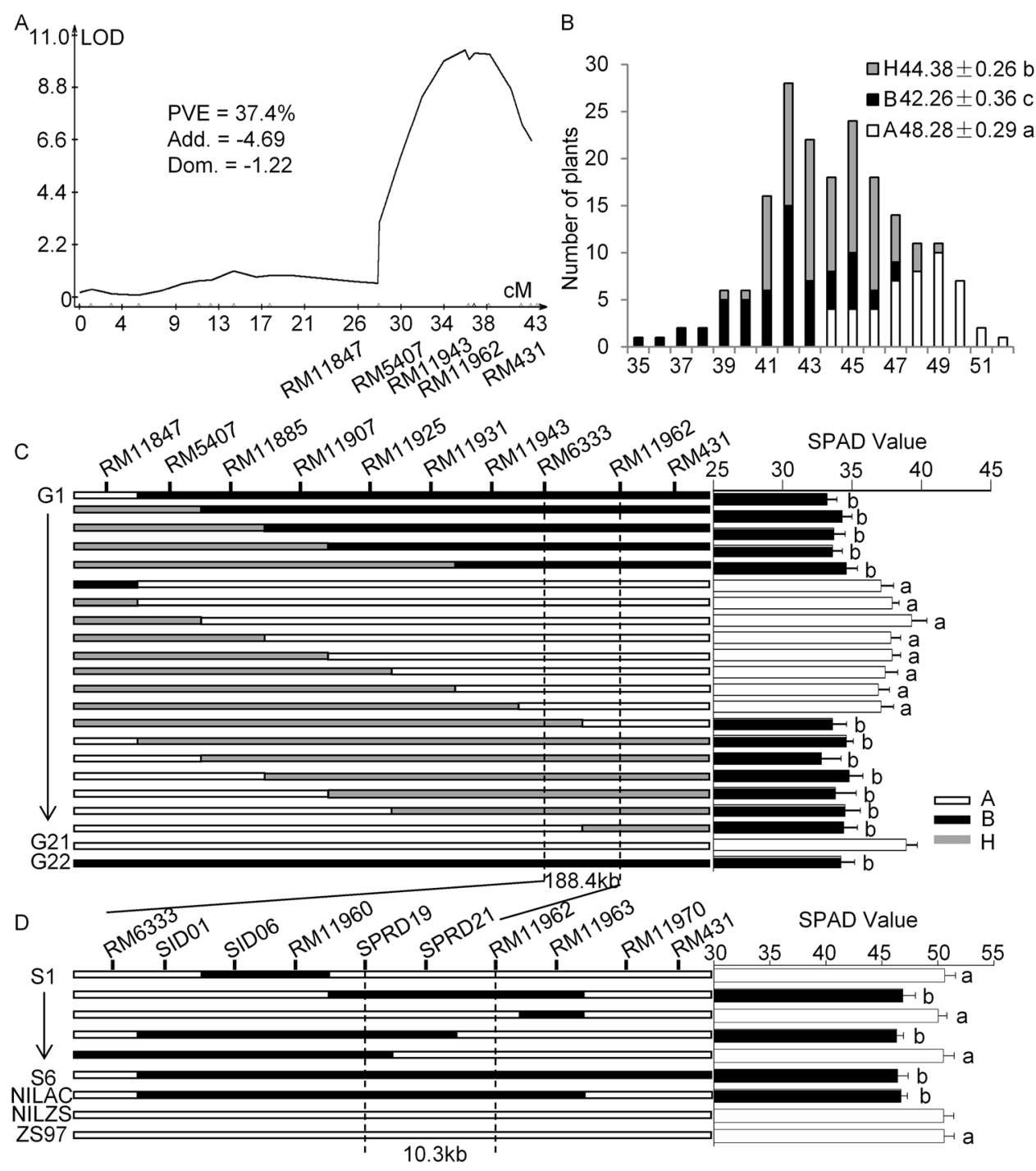

Figure 1. Detection and fine mapping of $q N C 1.2$ for leaf nitrogen content in rice. (A) Confirmation of the QTL for SPAD value using a CSSL-derived $\mathrm{F}_{2}$ population comprised of 190 individuals. PVE\% represents the phenotypic variance explained by the QTL; Add and Dom represent the additive and dominate effect, respectively. (B) Distribution of three genotypes in the population assayed by marker RM11943 that was tightly linked with the QTL. (C) Progeny testing of the recombinants delimited the QTL into a 188.4-kb interval. (D) Finely mapping of $q N C 1.2$ to a 10.3-kb region between RM6333 and RM11962. At right histogram, progeny testing of the recombinant lines with average SPAD value in flag leaves are indicated. Each bar represents mean $\pm S E(n=16)$. Different letters represent a significant difference at $P<0.01$ by Student's t-test between

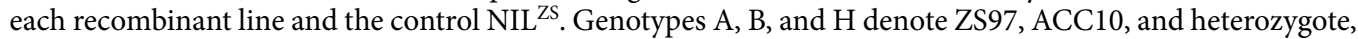
respectively.

Sequence analyses revealed that large variations (30 SNPs and InDels) occurred in the promoter and coding region of LOC_Os01g65780 of ACC10 and ZS97. In addition, the relative expression of this gene in several tissues, such as seedling leaf, stem, sheath, flag leaf, and panicle of $\mathrm{NIL}^{\mathrm{AC}}$ was significantly higher than that of NIL $^{\text {ZS }}$ (Figs. 2A, S3). Hence, LOC_Os01g65780 was the most likely candidate gene underlying $q N C 1.2$. Since LOC_Os01g65780, closest to GUX1 and GUX3 in Arabidopsis, displayed the conserved GT8 motif (Fig. S4A), we hereafter referred to it as OsGUX1.

OsGUX1 underlying qNC1.2 for chlorophyll content in leaf. To determine whether OsGUX1 affects leaf development, we constructed transgenic lines overexpressing OsGUX1 of ACC10 (OE780 $\left.{ }^{\mathrm{AC}}\right)$ in the ZS97 background. The transcript levels of the gene in three independent positive OE780 ${ }^{\mathrm{AC}}$ lines were approximately $100-450$ folds higher than those in the negative transgenic lines (Fig. S5A). The SPAD-value, chlorophyll $b$ content, and nitrogen content in flag leaves of positive OE780 ${ }^{\mathrm{AC}}$ lines were significantly lower than those of the negative lines (Fig. S5B-D).

Sequence analyses revealed that only one nucleotide variation was observed in the fourth exon of the candidate gene between the parental lines, leading to an amino acid change (V267M) at the $267^{\text {th }}$ site with valine (V) 


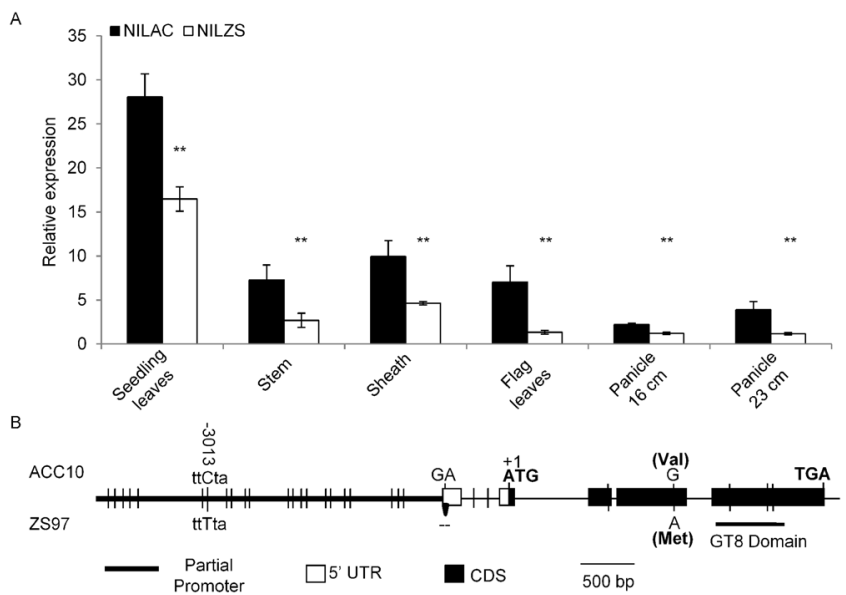

Figure 2. Expression profile and sequence variation of $O s G U X 1$ in the near isogenic lines (NILs). (A) qRT-PCR analysis of the expression of OsGUX1 relative to the geometric mean of three control genes (Ubiquitin, Actin1, $\beta$-tubulin) in various tissues from $\mathrm{NIL}^{\mathrm{AC}}$ and $\mathrm{NIL}^{\mathrm{ZS}}$. The experiment was conducted in biological triplicates with four technical replicates. Data are presented as means $\pm S E(n=3)$. The asterisks indicate significant differences between NIL ${ }^{\mathrm{AC}}$ and NIL ${ }^{\mathrm{ZS}}$ by student's t-test $(* \mathrm{P}<0.05, * * \mathrm{P}<0.01)$. (B,C) Schematic of the gene model showing allelic variation of OsGUX1 between ACC10 and ZS97. Arrow represents nucleotides deletion in the promoter region. Vertical line at a certain position indicates single nucleotide polymorphism between ACC10 and ZS97.

in ACC10 and methionine (M) in ZS97 (Fig. 2B). To investigate whether the variant V267M affects chlorophyll content, we isolated OsGUX1 from ZS97 to construct additional overexpression lines (OE780 ${ }^{\mathrm{ZS}}$ ). The transcript levels of the gene in three independent positive OE780 ${ }^{\mathrm{ZS}}$ lines were approximately 4-7 folds higher than those in the negative lines (Fig. 3A). Like OE780 ${ }^{\mathrm{AC}}$ lines did, the OE780 ${ }^{\mathrm{ZS}}$ lines decreased the SPAD-value, nitrogen content, and chlorophyll $b$ content in leaves compared with those in the corresponding negative lines (Fig. 3B-D). In contrast, the down-regulation of OsGUX1 expression in the positive RNAi lines (R780 ${ }^{\mathrm{ZS}}$ ) in the ZS97 background led to the significant increase in SPAD-value, chlorophyll $b$ and nitrogen content at flowering stage (Fig. 4A-D). These results indicate that functional differences between the ACC10 and ZS97 alleles might not be due to the single structural variation in encoding amino acids, but rather to the transcriptional change. Additionally, the SPAD-value and chlorophyll content were decreased in $\mathrm{OE780} 0^{\mathrm{ZS}}$ but they were increased in $\mathrm{R} 780^{\mathrm{ZS}}$ at seedling stage (Fig. S6), which was consistent with their variation tendency observed at flowering stage. Taken together, these results supports that $O s G U X 1$ is responsible for $q N C 1.2$.

Phylogenetic analysis and subcellular localization of OsGUX1. Using the predicted amino acids encoded by $O s G U X 1$, we retrieved 9 homologous protein sequences in Oryza sativa and 16 homologs in Arabidopsis thaliana. Phylogenetic analysis revealed that these homologous proteins fell into three clades, namely, GolS, GUXs, and IPUT1, respectively (Fig. S4A). OsGUX1 was grouped into the GUXs and was closest to GUX1 and GUX3 in Arabidopsis (Fig. S4A), which contained the conserved amino acid sequences including the DxD and HxxGxxKPW motifs identified previously ${ }^{23}$. These results indicate that OsGUX1 may have a similar function of GUX1 and GUX3 on cell wall components.

Subcellular localization analysis of the OsGUX1 fused with GFP revealed that OsGUX1 was localized to the Golgi apparatus in which xylan biosynthesis occurred. The single amino acid change (V267M) of OsGUX1 did not affect its localization in Golgi (Fig. S4B,C), which coincided with the localization of the homologous GUX1 and GUX2 that were previously reported as Golgi-localized glycosyltransferases in Arabidopsis ${ }^{17,24}$.

OsGUX1 affects cellular components and plant growth. Considering the effect of homologous genes GUX1 and GUX3 on cellular components in Arabidopsis ${ }^{19-21}$, we investigated several cellular components in the transgenic lines to determine whether OsGUX1 affected cellular components and plant development in rice. Positive R780 $\mathrm{ZS}$ lines markedly reduced the content of cellulose, hemicellulose, and starch in flag leaf, compared with the negative lines (Fig. $5 \mathrm{~A}-\mathrm{C}$ ). While positive $\mathrm{OE} 780^{\mathrm{ZS}}$ lines significantly increased the content of cellulose, hemicellulose and starch in flag leaf (Fig. 5D-F).

To further investigate whether OsGUX1 affected sugar substitutions on the xylan in rice leaf, we also used GC-MS (Gas Chromatography-mass Spectrometer) analysis to determine glucuronic acid (GlcA) and several monosaccharides including xylose, arabinose, and glucose in flag leaves of the OsGUX1 transgenic lines and NILs. The R780 $\mathrm{Zs}$ lines repressing OsGUX1 resulted in a significant decrease in GlcA, xylose, arabinose, and glucose content (Fig. 6A,D,G,J). Whereas the OE780 ${ }^{\mathrm{ZS}}$ lines overexpressing OsGUX1 caused an significant increase in GlcA and the three monosaccharides compared with the corresponding negative lines (Fig. 6B,E,H,K). The increased GlcA indicate that OsGUX1 has the function of adding glucuronic acid residues onto xylan. The increased xylose, arabinose and glucose suggest that xylan synthesis and hemicellulose linked glucan may be affected. These results indicate that OsGUX1 is involved in the xylan substitutions, leading to alteration of the cell wall depositions on rice leaf. 
A

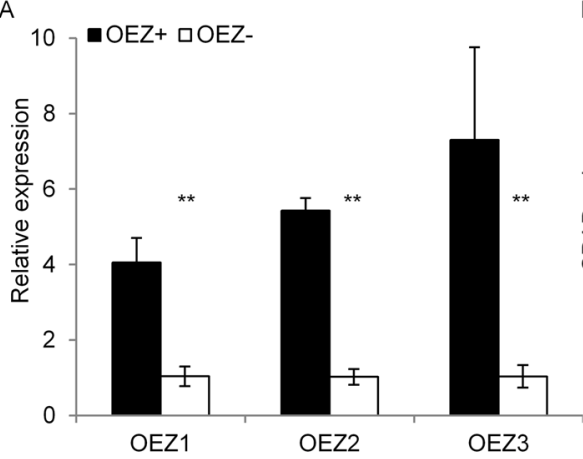

B

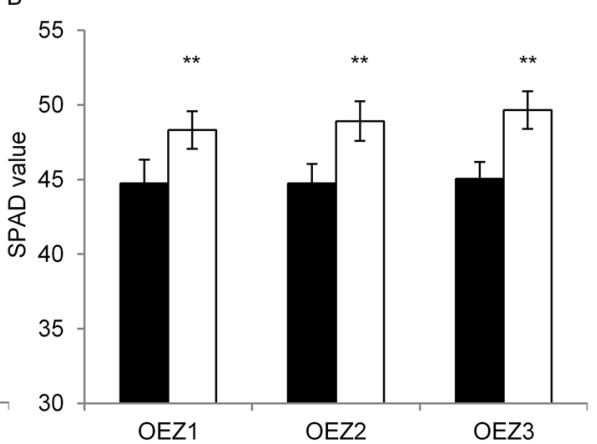

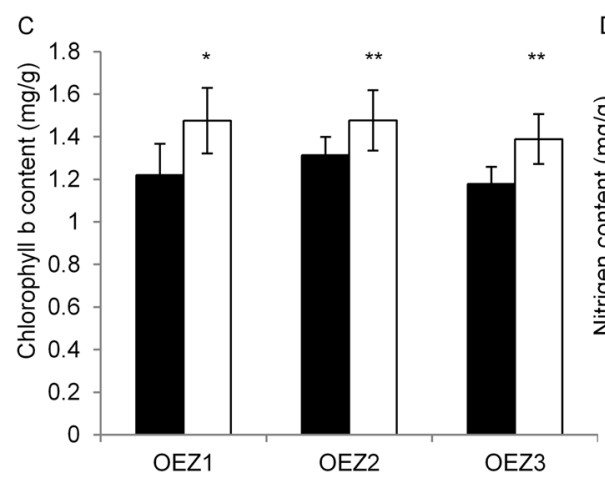

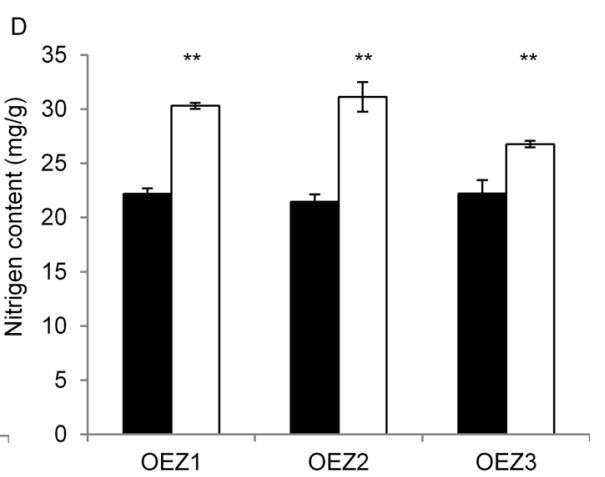

Figure 3. Relative expression and SPAD-related phenotype of flag leaves in three pairs of OsGUX1ZS overexpression lines (OEZ). (A) Relative expression of OsGUX1ZS. Data represent the mean $\pm \mathrm{SE}(\mathrm{n}=3)$. (B) SPAD value. (C) Chlorophyll $b$ content. (D) Nitrogen content. Asterisks indicate significant differences between the positive transgenic lines $(\mathrm{OEZ}+)$ and negative lines $(\mathrm{OEZ}-)$ by student's $\mathrm{t}$-test $(* \mathrm{P}<0.05$, $* * \mathrm{P}<0.01)$. Data represent the mean $\pm S E$ ( $n=8$ biological repeats) except that the mean of leaf nitrogen content is derived from three repeats.

In consistent with transgenic experiment results, NIL ${ }^{\mathrm{AC}}$ significantly decreased leaf nitrogen content (Fig. 7A,B), but significantly increased the contents of hemicellulose, cellulose, and starch compared with NIL ${ }^{\mathrm{ZS}}$ (Fig. 7C,D,E). The amounts of GlcA and the three monosaccharides in NIL ${ }^{\mathrm{AC}}$ was also significantly higher than that of NIL ${ }^{\text {ZS }}$ (Fig. 6C,F,I,L). Notably, R780 ${ }^{\text {ZS }}$ lines reduced seedling height and fresh weight (Fig. S7A,B). However, OE780 ${ }^{\mathrm{ZS}}$ lines promoted seedling growth (Fig. S7C,D). Consistently, the seedling weight and seedling height in NIL ${ }^{\mathrm{AC}}$ were significantly higher than those in NIL ${ }^{\mathrm{ZS}}$ (Fig. S8). In addition, NIL ${ }^{\mathrm{AC}}$ displayed an increased thousand grain weight and enhanced mechanical strength of the stems compared with NIL ${ }^{\text {ZS }}$ (Fig. 7K,L). These results suggest that OsGUX1 participates in accumulation of hemicellulose and cellulose, having strong effects on plant growth across various stages.

Stable difference in leaf nitrogen content between NILs. We conducted a dynamic investigation of leaf nitrogen content in the NILs at different time points from day 6 before heading to 13 day after heading (DAH) (Fig. S9A). A peak of SPAD-value appeared on $7 \mathrm{DAH}$, the value began to decline gradually from $7 \mathrm{DAH}$. The net photosynthetic rate of NIL ${ }^{\mathrm{AC}}$ significantly reduced, compared with that of NIL ${ }^{\mathrm{ZS}}$ (Fig. 7G), which was consistent with the observation of an decreased total chlorophyll content in flag leaves. However, no difference in the stomatal conductance, intercellular $\mathrm{CO}_{2}$ concentration and transpiration rate was observed between $\mathrm{NIL}^{\mathrm{AC}}$ and NIL ${ }^{\mathrm{ZS}}$ at heading stage (Fig. $7 \mathrm{H}-\mathrm{J})$.

To determine whether the expression level of OsGUX1 was affected by nitrogen application, we conducted hydroponic experiments with NILs at seedling stage under three nitrogen gradients. The results revealed that relative expression level of OsGUX1 was induced with the increase of nitrogen application in both NIL ${ }^{\mathrm{AC}}$ and $\mathrm{NIL}^{\mathrm{ZS}}$, and that the expression level of OsGUX1 in $\mathrm{NIL}^{\mathrm{AC}}$ was significantly higher than that in $\mathrm{NIL}^{\mathrm{ZS}}$ under all three nitrogen gradients (Fig. S9B). Particularly, under low nitrogen level, the expression of OsGUX1 in NIL ${ }^{\mathrm{AC}}$ was almost two times as high as that in NIL ${ }^{\mathrm{ZS}}$. However, the relative expression of OsGUX1 was slightly inhibited under high nitrogen level in both NILs compared with that under normal nitrogen application. As a result, the SPAD-value, nitrogen content, and chlorophyll $b$ in NIL ${ }^{A C}$ were significantly lower than those in NIL ${ }^{\mathrm{ZS}}$ under all nitrogen levels (Fig. S9), although a significant increase in the three assayed traits were observed between low and normal nitrogen level for the same NIL. These results indicated that OsGUX1 associated with the leaf N content was independent of exterior $\mathrm{N}$ application.

Thickened primary cell wall may affect relative chlorophyll content. It has been reported that the linear relationship between SPAD value and leaf $\mathrm{N}$ concentration varies with crop growth stage and variety, mostly due to leaf thickness ${ }^{25}$. Thus, we compared the size or area of large vascular bundle (LVB) and small 

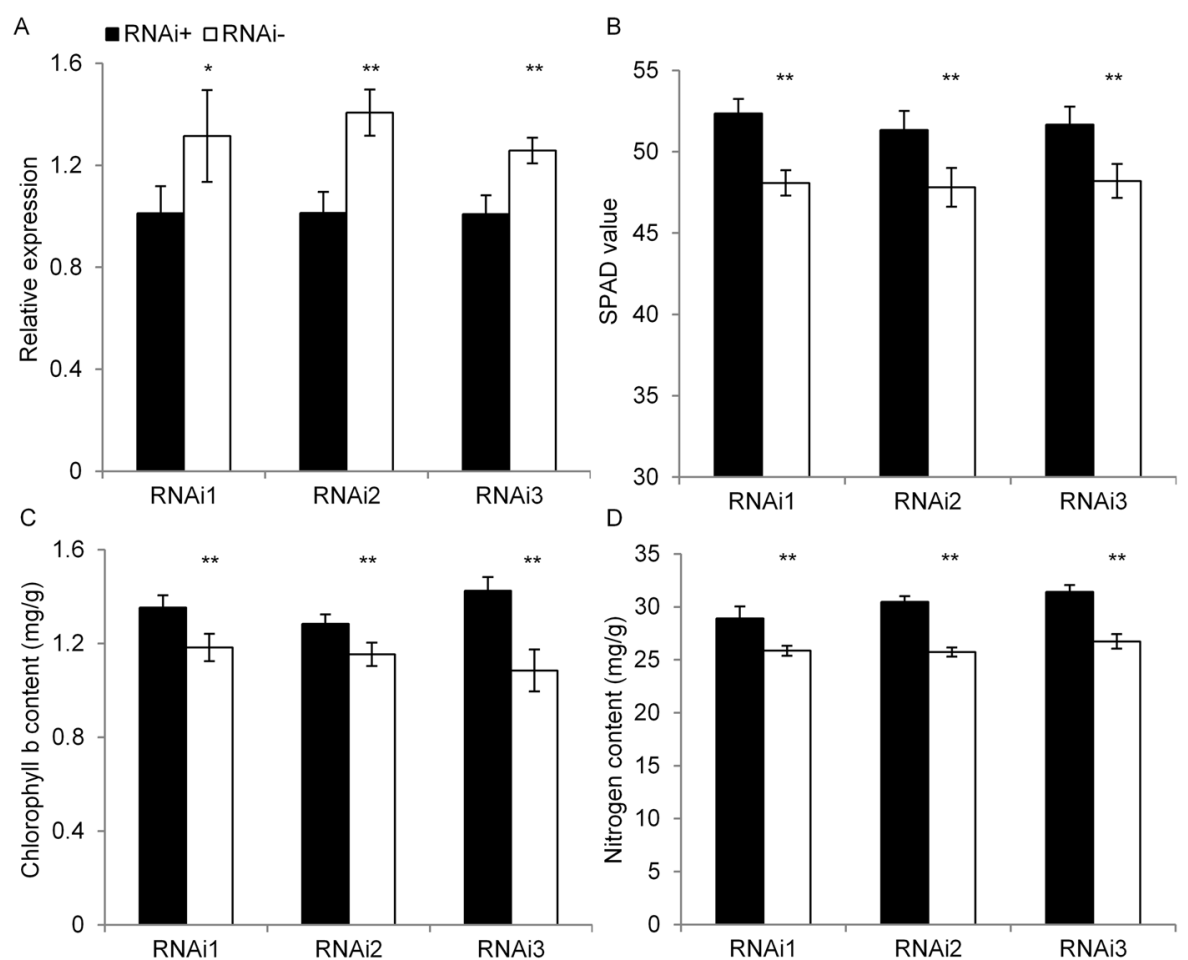

Figure 4. Relative expression and SPAD-related phenotype of flag leaves in three pairs of $O s G U X 1^{Z S}$ RNAi lines (RNAi 1-3). (A) Relative expression of OsGUX1 ${ }^{Z S}$. (B) SPAD value. (C) Content of chlorophyll $b$. (D) Content of nitrogen. Asterisks indicate significant differences between the positive lines (RNAi+, black) and negative lines (RNAi-, white) by student's t-test $(* \mathrm{P}<0.05$, $* * \mathrm{P}<0.01)$. The errors bar in $(\mathbf{B}, \mathbf{C})$ represent the mean $\pm \mathrm{SE}$ $(\mathrm{n}=8)$, data in $(\mathbf{A}, \mathbf{D})$ is derived from three repeats.
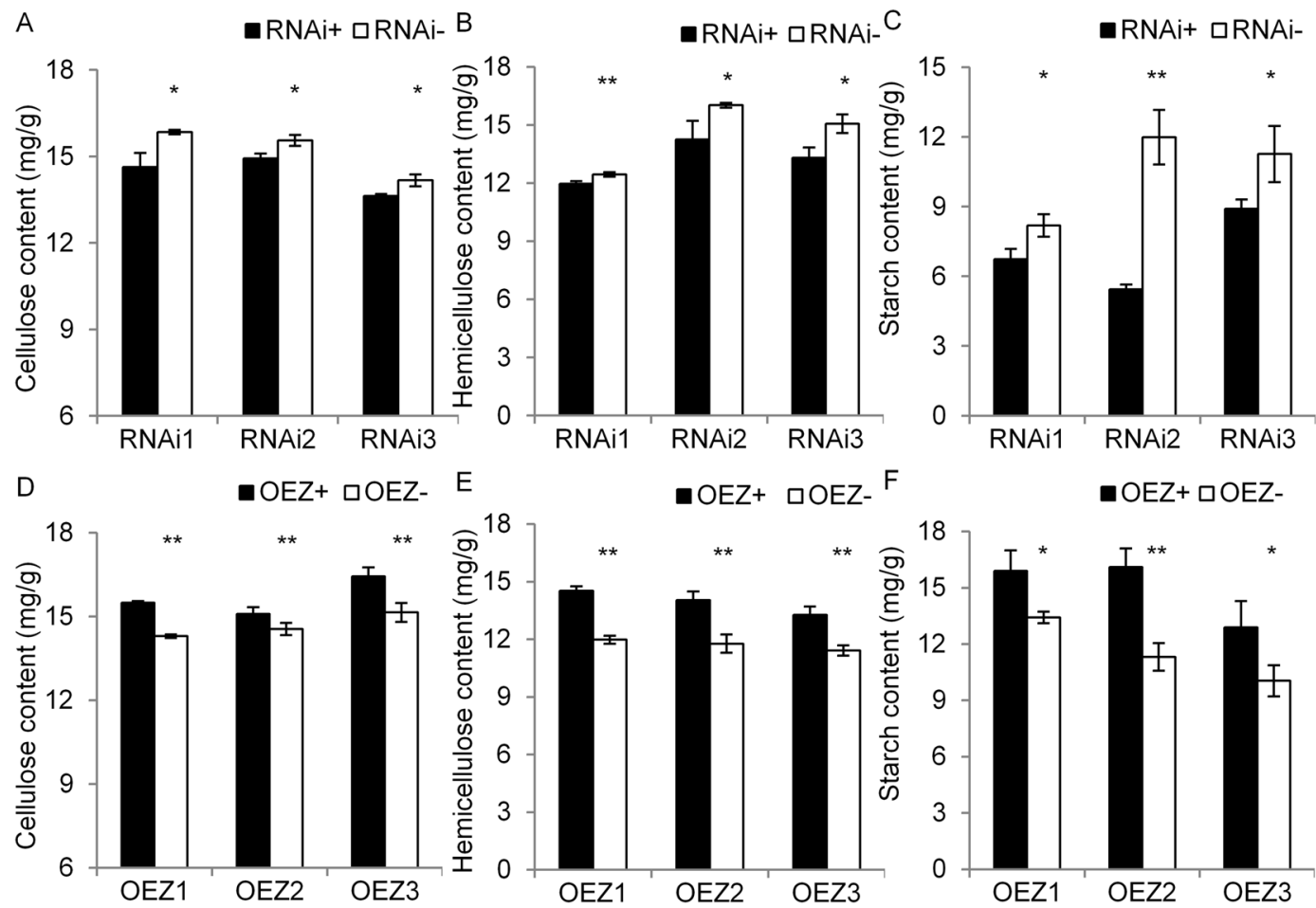

Figure 5. Cell components of flag leaves in $O s G U X 1^{Z S}$ RNAi and overexpression (OEZ) lines. (A,D) Cellulose content. (B,E) Hemicellulose content. (C,F) Starch content. Data represent the mean $\pm \mathrm{SE}$ ( $\mathrm{n}=3$, with eight leaf samples mixed for each repeat). The asterisks indicate significant differences between the positive $(+)$ and negative $(-)$ lines by student's t-test $(* \mathrm{P}<0.05, * * \mathrm{P}<0.01)$. 

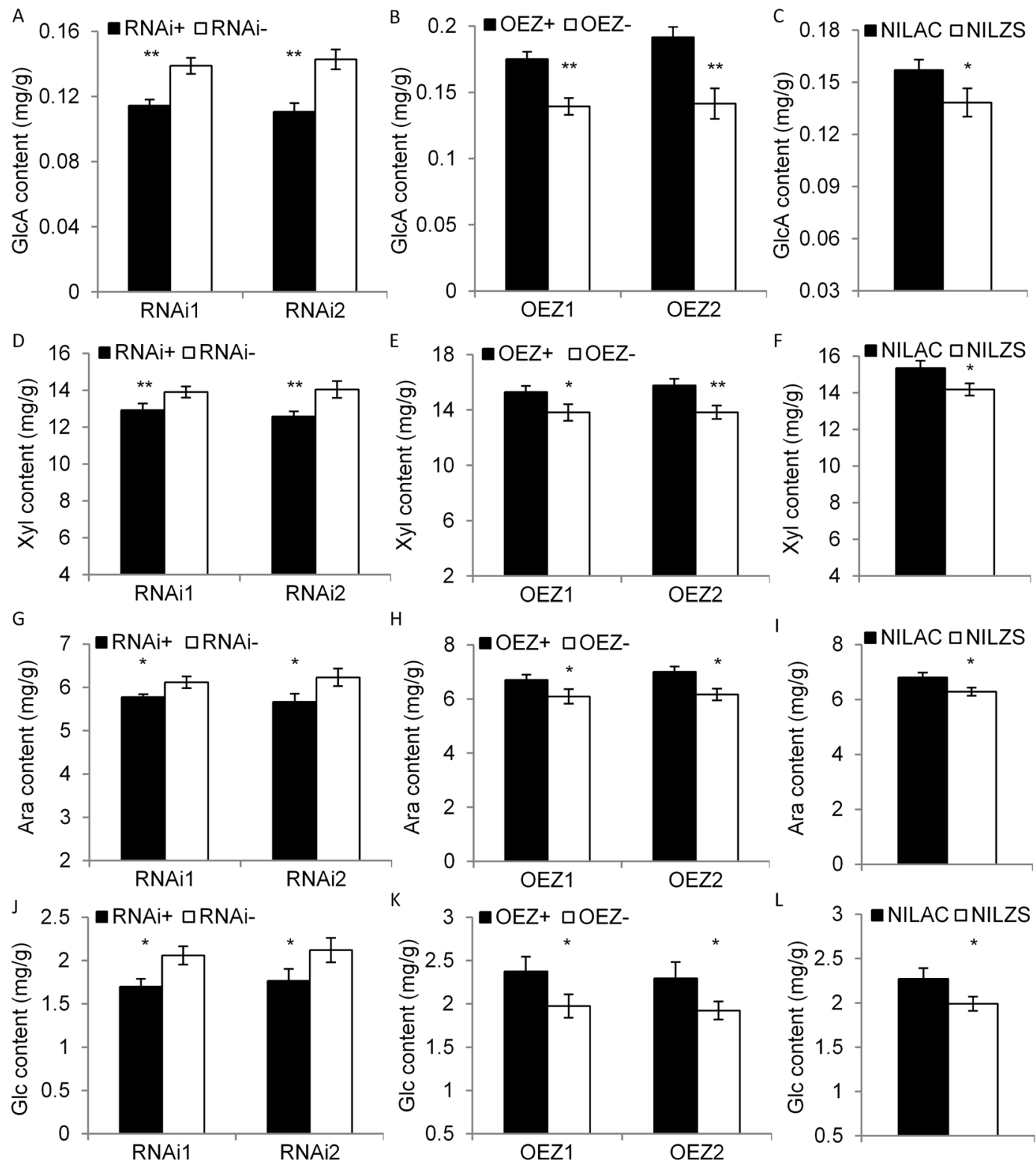

Figure 6. Monosaccharide analysis of flag leaves in OsGUX1 ${ }^{Z S} \mathrm{RNAi}$, overexpression (OEZ) lines and NILs. (A-C) Glucuronic acid (GlcA). (D-F) Xylose (Xyl). (G-I) Arabinose (Ara). (J-L) Glucose (Glc). The asterisks indicate significant differences between the pairwise positive $(+)$ and negative $(-)$ lines and in NILs by student's t-test $(* \mathrm{P}<0.05, * * \mathrm{P}<0.01)$. Data represent the mean $\pm \mathrm{SE}(\mathrm{n}=4$, with mixed eight leaf samples per repeat).

vascular bundle (SVB) through cross-sections of flag leaves of NILs, which represented the thickness of leaves (Fig. 8A,B). The histological section analysis revealed no difference in the average size of LVB between NIL ${ }^{A C}$ and NIL $^{\text {ZS }}$ (Fig. 8C), while the thickness of SVB of NIL ${ }^{\mathrm{AC}}$ was significantly larger than that of NIL ${ }^{\mathrm{ZS}}$ (Fig. 8D), but the length between adjacent SVBs of $\mathrm{NIL}^{\mathrm{AC}}$ was shorter than that of NIL ${ }^{\mathrm{ZS}}$ (Fig. 8E). Consequently, no difference in the cross-sectional area containing mesophyll cells was observed between NILs (Fig. 8F). Intriguingly, the observation of the mesophyll cells by a transmission electron microscope revealed that the primary cell wall of mesophyll cells in $\mathrm{NIL}^{\mathrm{AC}}$ was significantly thicker than that in NIL ${ }^{\mathrm{ZS}}$ (Fig. 8G,H). These results suggest that primary cell wall of mesophyll cells might affect relative chlorophyll content in leaf.

Expression of xylan and cellulose biosynthesis-related genes. Because cellulose and hemicellulose contents were increased in the overexpression OsGUX1 transgenic lines and NIL ${ }^{\mathrm{AC}}$, we used quantitative RT-PCR to analyze transcript levels of several key genes related to the wall polymers syntheses to explore whether OsGUX1 affects xylan and cellulose syntheses. The results reveal that the transcript levels of eight biosynthetic genes involved xylan synthesis (OsIRX9L and OsIRX14) and cellulose synthases (OsCesA1, OsCesA2, OsCesA3, OsCesA5, OsCesA6, OsCesA8) in $\mathrm{NIL}^{\mathrm{AC}}$ were significantly higher than that of $\mathrm{NIL}^{\mathrm{ZS}}$ (Fig. S10A,B). It has been reported that these cellulose-related genes are strongly co-expressed in seedlings and other typical young 

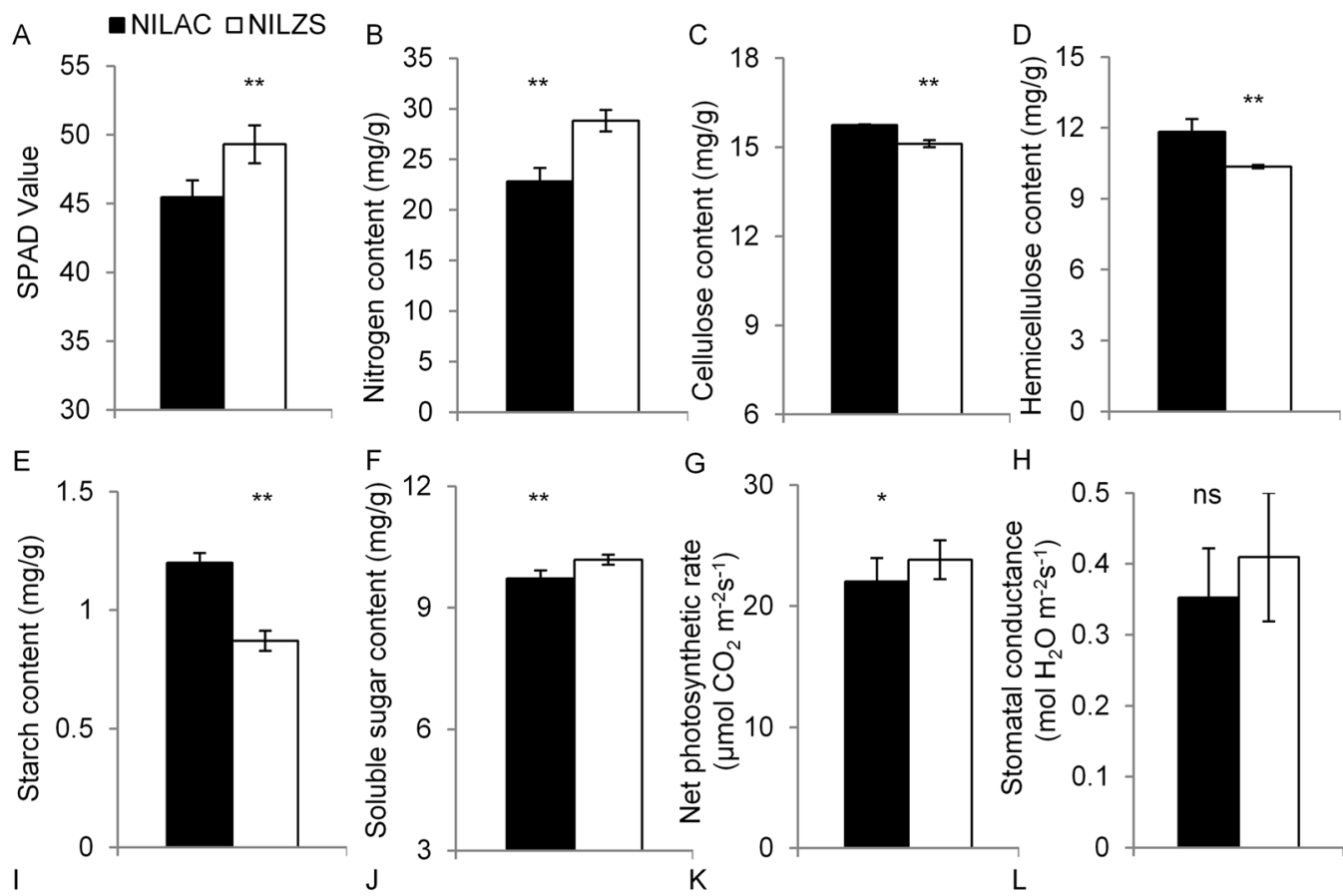

$\mathrm{H}$
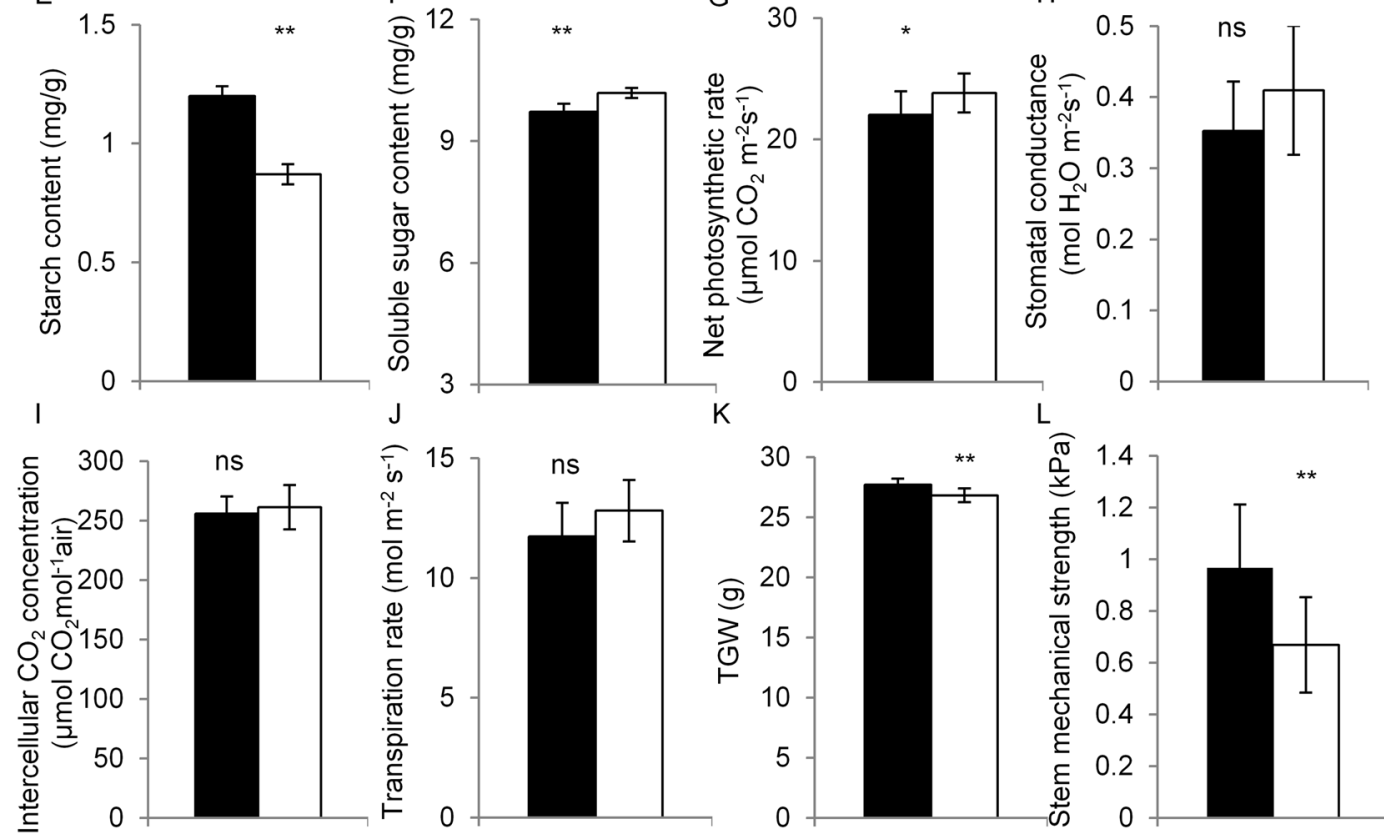

Figure 7. Phenotype investigation in $\mathrm{NIL}^{\mathrm{AC}}$ and $\mathrm{NIL}^{\mathrm{ZS}}$ on day 7 after heading. (A) SPAD value. (B) Nitrogen content. (C) Cellulose content. (D) Hemicellulose content. (E) Starch content. (F) Soluble sugar content. (G) Net photosynthetic rate. (H) Stomatal conductance. (I) Intercellular $\mathrm{CO}_{2}$ concentration. (J) Transpiration rate. (K) Thousand grain weight. (L) Mechanical strength of stems. The asterisks indicate significant differences between $\mathrm{NIL}^{\mathrm{AC}}$ and NIL ${ }^{\mathrm{ZS}}$ by student's t-test $(* \mathrm{P}<0.05$, $* * \mathrm{P}<0.01$, ns, non significant). Data represent the mean $\pm S E(n=3$, with at least eight leaf samples mixed per repeat).

vegetative tissues rich in primary cell walls ${ }^{26}$, and that $O s I R X 9 L$ and $O s I R X 14$ are involved in the construction of xylan backbones in primary and secondary cell walls ${ }^{27}$. The elevated expression of xylan synthesis-related genes and cellulose synthase genes in $\mathrm{NIL}^{\mathrm{AC}}$ was consistent with the increased cellulose, hemicellulose levels in leaves described above. These results further suggest that OsGUX1 participates in the processes of xylan and cellulose biosynthesis.

Interestingly, lower soluble sugar content in $\mathrm{NIL}^{\mathrm{AC}}$ was observed than those in NIL ${ }^{\mathrm{ZS}}$ (Fig. 7F). We then analyzed the expression levels of several sucrose-related genes and UDP-Glc in flag leaves of both NILs at the 7 DAH. These genes have been reported to be involved in the carbon supply during cell wall synthesis ${ }^{28}$. The quantitative RT-PCR results indicate that the assayed sucrose-related genes were significantly induced in NIL ${ }^{\mathrm{AC}}$, compared with those in NIL ${ }^{\mathrm{ZS}}$ (Fig. S10C).

It is notable that PHD1 (Photoassimilate Defective 1) was significantly repressed in $\mathrm{NIL}^{\mathrm{AC}}$ compared with that in NIL ${ }^{\text {ZS }}$ (Fig. S10C). Previous study reported that PHD1 encoding a chloroplast-localized UDP-Glc epimerase was responsible for the biosynthesis of UDP-galactose in chloroplast, and that PHD1 played an important role in supplying sufficient galactolipids to thylakoid membranes for chloroplast function and photosynthetic activity ${ }^{29}$. The phd1 mutant decreased chlorophyll content, photosynthetic activity, and altered chloroplast ultrastructure. Thus, OsGUX1 might play a role in affecting the assignment of carbonhydrate sources and the increased use of UDP-Glc for the cell wall biosynthesis.

\section{Discussion}

This present study reports the cloning and identification of the xylan glucuronosyltransferase gene OsGUX1 that affects relative chlorophyll content in rice. Leaf chlorophyll content is an important physiological indicator of plant stress ${ }^{30}$, senescence ${ }^{31}$, and nitrogen content ${ }^{32}$. However, the genetic linkage between chlorophyll content and leaf structure remains largely unknown. As a first step toward dissecting the genetic bases of such complex 


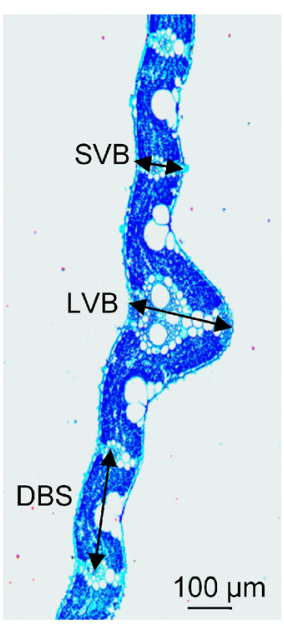

NILAC

C

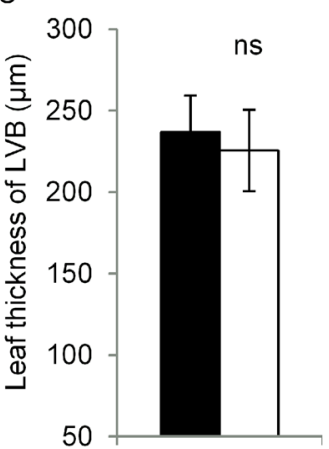

B

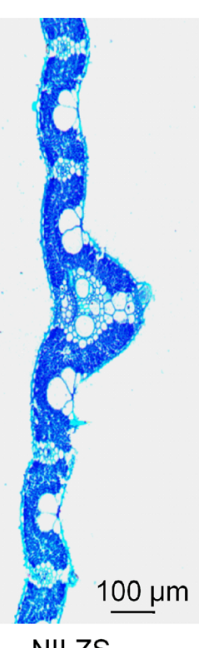

NILZS

G
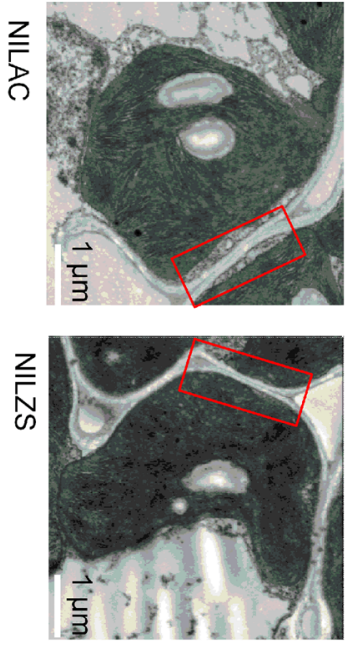

$\mathrm{H}$

D

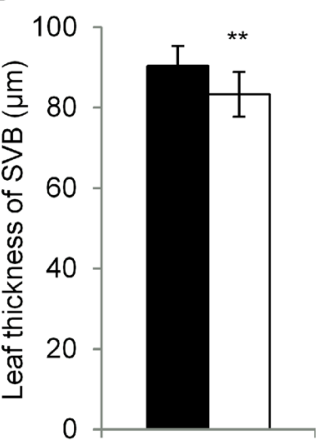

E

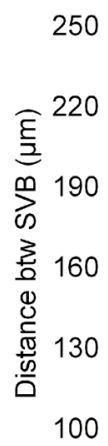

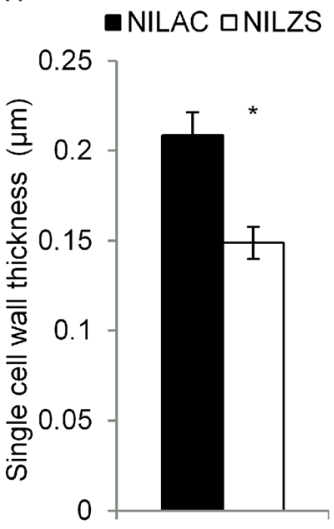

$\mathrm{F}$

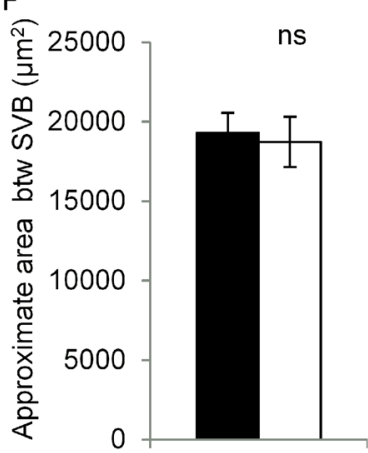

Figure 8. Leaf anatomical characteristics and cell wall thickness in NILs. (A,B) Cross-sections of flag leaves stained with toluidine blue, (A) NIL ${ }^{\mathrm{AC}}$; (B) NIL ${ }^{\mathrm{ZS}}$. (C,D) Comparison of thickness of flag leaves in the NILs. (C) Leaf thickness of LVB (large vascular bundle); (D) Leaf thickness of SVB (small vascular bundle). (E) Distance between adjacent SVBs. (F) Approximate area within adjacent SVBs $\left(\mu \mathrm{m}^{2}\right)$. (G) Transmission electron microscopic observation of mesophyll cell walls in the NILs. (H) Cell wall thickness in the NILs. Data represent the mean $\pm S E(n=5$, with 15 leaf sections harvested from 5 plants for each replicate). The asterisks indicate significant differences between $\mathrm{NIL}^{\mathrm{AC}}$ and $\mathrm{NIL}^{\mathrm{ZS}}$ by student's t-test $(* \mathrm{P}<0.05, * * \mathrm{P}<0.01)$.

trait, we used a SPAD chlorophyll meter to explore the key factors related to relative chlorophyll content in flag leaves in the developed CSSL population from the cross of ACC10 and ZS97 (Fig. S1). We have identified at least four loci for leaf chlorophyll content (Table S2). Of them, three loci are localized to the same or nearby regions for chlorophyll content as reported in previous studies in rice ${ }^{33-39}$. For example, $q N C 1.1$ in our study was found in the same region related to chlorophyll content reported in other studies ${ }^{36,37} . q N C 7.1$ was close to the QTL detected in the reports ${ }^{38,39}$. $q N C 10.1$ was mapped to the same location reported previously ${ }^{36,40}$. This co-localization supports the accuracy of the QTL analysis of leaf chlorophyll content using the CSSL population. As a major locus $q N C 1.2$ is a newly detected for leaf chlorophyll content, we thus using a map-based cloning strategy to identify the gene underlying $q N C 1.2$, and have defined $O s G U X 1$ as the candidate gene for leaf chlorophyll content (Fig. 1). The allele OsGUX $1^{A C}$ from a wild rice ACC10 significantly decreased nitrogen content and chlorophyll content of leaf, resulting in a reduction in photosynthetic rate of leaf compared with $O s G U X 1^{Z S}$. The transgenic lines overexpressing OsGUX1 decreased relative chlorophyll content measured as SPAD-value, chlorophyll $a$, chlorophyll $b$, and carotenoid content in leaves at seedling stage (Fig. S6K-N), and also reduced the contents of chlorophyll in flag leaves at heading stage (Fig. 3B-D). While, the transgenic lines suppressing OsGUX1 increased the contents of chlorophyll and nitrogen in leaves (Fig. 4B-D). It is notable that the expression patterns of OsGUX2 and $O s G U X 3$, two close homologous genes of OsGUX1, are different from that of OsGUX1 (Figs. 2A and S10A,B). OsGUX2 (LOC_Os02g35020.1) has high expression in flag leaves and low expression in stems, while OsGUX3 (LOC_Os03g08600.1) has high expression in stems and low expression in leaves (Fig. S11). However, there are no significant differences in the expression of OsGUX2 and OsGUX3 in NIL ${ }^{\mathrm{AC}}$ compared with that of NIL ${ }^{\mathrm{ZS}}$. Furthermore, the expression of these two homologs was not altered in the OsGUX1-RNAi lines (Fig. S11C,D), suggesting that significant difference in leaf chlorophyll content in the OsGUX1-RNAi lines and NILs did not result from $O s G U X 2$ and $O s G U X 3$. Collectively, overexpression and suppression of OsGUX1 experiments and NIL assays indicate that OsGUX1 expression level affects leaf chlorophyll content in rice. This identification of the major locus like $q N C 1.2$ provides an opportunity to elucidate the relationship of the cell wall properties and chlorophyll content of leaf. 
Our experimental data support that $O s G U X 1$ plays a role in adding glucuronic acid residues onto xylan, which affecting hemicellulose and cellulose levels of the leaf primary cell wall in rice. Firstly, OsGUX1 has low expression in stems and high expression in leaves (Figs. 2A and S3), suggesting that it is involved in primary wall biosynthesis. Secondly overexpression of OsGUX1 lines and NIL ${ }^{\mathrm{AC}}$ exhibited higher levels of GlcA, xylose, arabinose, and glucose in flag leaves than their counterparts. In contrast, suppression of OsGUX1 lines revealed the opposite effect on GlcA and the three monosaccharides (Fig. 6). These results provide strong evidence that OsGUX1 is involved in sugar substitutions on xylan. It is expected that the sugar substitutions on the primary cell wall xylan may influence the amount and structure of hemicelluloses, further affecting other cell wall components. Thirdly, our results have revealed that higher transcript levels of xylan synthesis-related genes and cellulose synthase genes were induced in overexpression OsGUX1 lines, and lower transcript levels of these synthesis genes in suppression of OsGUX1 lines than the corresponding negative lines (Fig. S10). Consistently, overexpression of OsGUX1 increased the content of cellulose and hemicellulose, while suppression of OsGUX1 decreased the content of cellulose and hemicellulose in rice leaf (Fig. 5). In addition, the contents of cellulose and hemicellulose in NIL ${ }^{\mathrm{AC}}$ were significantly higher than those in NIL ${ }^{\mathrm{ZS}}$. It has been reported that GUX3 in Arabidopsis, a closely homologous gene to OsGUX1 (Fig. S4), was highly expressed in the tissues in which primary cell wall synthesis occurring, and that GUX3 is required for the addition of the GlcA decorations on the primary cell wall xylan and affected content of cellulose and hemicellulose ${ }^{20,21}$. Thus, the current data collectively suggest that OsGUX1 has the function of transferring GlcA onto xylan, which affects the compositions of the primary cell wall in rice.

It is notable that OsGUX1 could alter seedling growth and the mechanical strength of stems in rice (Figs. 7L and S7). In Arabidopisis, both GUX1 and GUX2 are responsible for the substitution of the xylan backbone with $[\mathrm{Me}] \mathrm{GlcA}$ in the secondary cell wall ${ }^{19,21,41}$. The complete disappearance of secondary wall xylan GlcA and MeGlcA side chain in the triple mutant guxlgux2gux3 had no impact on the xylem vessels and plant growth ${ }^{20}$. Hence, characterization of OsGUX1 provides new knowledge about the role of GlcA substitutions in plant growth appear to be different from that in dicots.

The most noteworthy finding of the present study is that OsGUX1 involves in xylan substitutions in the primary cell wall affecting leaf chlorophyll content. However, it is an open question how OsGUX1 for xylan substitution plays an important role in leaf chlorophyll content. We propose that OsGUX1 may affect cell wall compositions in the mesophyll cells, which indirectly influence the chlorophyll content. Our experimental data indicate that OsGUX1 for the GlcA substitution of xylan in leaf has a strong effect on cell wall thickness of mesophyll cells, which may result in leaf development as evidenced by changes in nitrogen and chlorophyll. Several lines of evidence support this note. Firstly, a transmission electron microscope analysis revealed that the primary cell wall of mesophyll cells in NIL ${ }^{\text {AC }}$ was significantly thicker than that in NIL ${ }^{\mathrm{ZS}}$ (Fig. 8G,H). Secondly, high expression of OsGUX1 induced the expressions of xylan synthesis genes, which leads to the accumulation of cellulose and hemicelluloses (Figs. 7 and S10), thus thickening the primary cell walls. The increased cell wall thickness may be the additional contributor to mechanical strength (Fig. 7L). In addition, the thicker walls could cause decreased soluble sugars in leaf. In agreement with this case, lower soluble sugar level in NIL ${ }^{\mathrm{AC}}$ was observed than that in NIL ${ }^{\mathrm{ZS}}$ (Fig. 7F). As a response to the decrease of soluble sugar content, the expression levels of several genes related to the sucrose decomposition, transport, and metabolism in rice leaf were induced (Fig. S10C). It is also possible that the accumulation of cell wall components increased use of UDP-Glc, which may affect the UDP-galactose biosynthesis in chloroplast (Fig. S10C), leading to altered chloroplast ultrastructure and chlorophyll content. Therefore, OsGUX1 thickened primary cell wall of mesophyll cells results in the decline in chlorophyll content of leaves, although the related genes participate in the chloroplast development process remains to be further investigated. Because chlorophyll content and nitrogen content of leaf is highly associated with photosynthesis and plant growth, discovery of the OsGUX1 functions on primary cell wall components and leaf chlorophyll content will provide an option to improving the potential rice yield by adjusting leaf nitrogen status and enhancing the mechanical strength of stems under different nitrogen application conditions.

\section{Materials and Methods}

CSSL population and genotyping. The chromosomal segment substitution lines population was developed by a backcross scheme coupled with marker-aided selection. In this population, an O. rufipogon (accession: IRGC105491, ACC10) as donor parent was crossed with ZS97 as receptor parent, followed by six-time backcross (Fig. S1). A set of 111 lines, designated as CSSL1 to CSSL111, were used for phenotype collection and QTL detection (Fig. S2). The CSSL population was previously genotyped using 176 simple sequence repeat (SSR) markers on the published linkage map of rice (http://www.gramene.org). The results showed that each line harbored one or a few substituted ACC10 segments at particular chromosomal region in the genetic background of ZS97 $7^{42}$. To determine the genotype precisely, a high-throughput Infinium RICE6K array (Illumina) containing 5102 SNP markers evenly distributed on the 12 rice chromosomes with an average density of 12 SNPs per Mb, was used to analyze the genotypes of CSSLs ${ }^{22}$.

The CSSL population, along with the parental lines ACC10 and ZS97, was grown in a three-row plot for each line with 10 individuals per row at a spacing of $16.7 \mathrm{~cm} \times 26.6 \mathrm{~cm}$ in the experimental field of Huazhong Agricultural University $\left(30.4^{\circ} \mathrm{N}, 114.2^{\circ} \mathrm{E}\right)$. The experiments followed a randomized block design with two replications for each line.

Bin mapping of QTL. On basis of SNP genotyping, a bin was defined by a unique overlapping substitution segment from the CSSL population as described previously ${ }^{43}$. QTL analysis was conducted in the CSSL population with the bins as markers by the composite interval mapping method in the software IciMapping v4.0 (http:// www.isbreeding.net/software/). QTL nomenclature followed the principle suggested by McCouch and CGSNL ${ }^{44}$.

DNA extract and PCR reaction. DNA of the CSSLs and derived lines was extracted using the CTAB $\operatorname{method}^{45}$. The PCR reaction mixture consisted of $2 \mu \mathrm{l}$ template $(50 \mathrm{ng} / \mu \mathrm{l}), 2 \mu \mathrm{l} 10 \times$ PCR Buffer $\left(\mathrm{Mg}^{2+}\right.$ free $), 1.6 \mu \mathrm{l}$ 


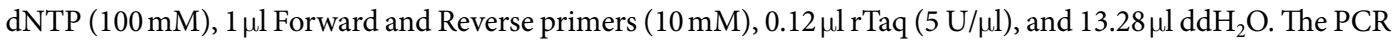
reaction was performed at $94^{\circ} \mathrm{C}$ for denaturation $5 \mathrm{~min}$, and then for $35 \mathrm{cycles}$ of $94^{\circ} \mathrm{C}$ for $30 \mathrm{sec}, 55^{\circ} \mathrm{C}$ for $30 \mathrm{sec}$, followed by $72^{\circ} \mathrm{C}$ for $5 \mathrm{~min}$, and ended with $25^{\circ} \mathrm{C}$ for $1 \mathrm{~min}$.

Data analysis in $F_{2}$ population and progeny testing. QTL analyses in the CSSL-derived segregating population were performed using WinQTLcart $2.5^{46}$. Logarithm of the odds (LOD) score for declaring the presence of QTL for each trait was estimated by 1,000 permutations ${ }^{47}$. As a result of the permutation, an average threshold of 2.5 was used for a QTL declaration for each trait. Progeny testing of homozygous recombinant lines was conducted for precisely phenotyping. In the process of fine-mapping, two near isogenic lines (NILs) at the target QTL region were developed from a heterozygote of CSSL containing a small introduced target segment for the phenotypic characterization.

RNA extract and quantitative real-time PCR analyses. Total RNA was isolated from the tissues using a RNA extraction kit (TRIzol reagent, Invitrogen) according to the manufacturer's instructions. The cDNA was obtained from approximate $3 \mu \mathrm{g}$ of total RNA using M-MLV First Strand Kit (Invitrogen, China). Quantitative RT-PCR was carried out in a total volume of $10 \mu \mathrm{l}$ containing $4.6 \mu \mathrm{l}$ of cDNA, $0.2 \mu \mathrm{M}$ of primer mix, and $5 \mu \mathrm{l}$ SYBR Green Master Mix (Roche) on a real-time PCR system StepOne ${ }^{\text {Plus }}$ (Applied Biosystems). Three rice genes Ubiquitin (LOC_Os03g13170), Actin1 (LOC_Os03g50885) and $\beta$-tubulin (LOC_Os01g59150) were used as the internal control for all expression analyses. As similar expression results were observed regardless of which control genes used, a geometric mean of expressions of the three control genes was applied for the relative expression analyses for every assayed sample as described previously ${ }^{48}$. The assay was carried out with three biological replicates, and each replicate contained four technical repeats. The relative quantification of expression was based on the $2^{-\Delta \Delta C T}$ method $^{49}$. All qRT-PCR primers are listed in the Supplementary Table S3. The sequences for all assayed genes in this study can be found in the Rice Genome Annotation Project website (http://rice.plantbiology. msu.edu/).

Vector construction and rice transformation. To construct overexpression vectors for OsGUX1, the cDNA of OsGUX1 from ZS97 and ACC10 was respectively amplified with the primers PU7804F and PU7804R, and cloned into T-vector (Promega). The sequence-confirmed clones containing OsGUX1 were digested by Sac I and Pst I, and followed by the insertion of OsGUX1 into the pCAMBIA1301S vector with the 35S promoter to produce the fusion vector 35S:OsGUX1.

To construct an RNAi vector for OsGUX1, a 403-bp fragment of OsGUX1 was amplified from ZS97 cDNA with the primers PDS780F and PDS780R. And then this 403-bp fragment was inserted in the forward and reverse directions into pDS1301 vector, a modified version of pCAMBIA1301 ${ }^{50}$. The overexpression vectors and RNAi suppression vectors were independently introduced into ZS97 by Agrobacterium-mediated transformation as described previously ${ }^{51}$. The transgenic rice plants were confirmed by PCR using specific primers (Table S3).

Subcellular localization of OsGUX1 protein. For investigating the subcellular localization of the OsGUX1 $1^{\mathrm{AC}}$ and OsGUX1 ${ }^{\mathrm{ZS}}$ protein, the coding region of $O s G U X 1^{A C}$ and $O s G U X 1^{Z S}$ was cloned into the PM999-35S vector fused with C-terminal green fluorescent protein (GFP), respectively. The resultant construct (OsGUX1 ${ }^{\mathrm{AC}}-\mathrm{GFP}$ or OsGUX1 ${ }^{\mathrm{ZS}}-\mathrm{GFP}$ ) and the Golgi marker Man1-RFP (red fluorescent protein) were transiently expressed in rice protoplasts as described previously ${ }^{52,53}$. The fluorescence signal was observed with a confocal laser scanning microscope (Leica FV1200, Olympus) after the transformed cells were incubated in darkness at $28^{\circ} \mathrm{C}$ for $16-18 \mathrm{~h}$, with excitation wavelengths of $488 \mathrm{~nm}$ and $561 \mathrm{~nm}$ for GFP and RFP, respectively.

Protein sequence of multiple alignment. The protein sequences of OsGUX1 $1^{\mathrm{ZS}}$ and OsGUX1 $1^{\mathrm{AC}}$ were aligned with the homologous proteins that were retrieved from Phytozome v12.1 database ${ }^{54}$. The multiple sequence alignment of the full-length proteins was performed in the complete alignment method by the ClustalX in MEGA $6^{55}$. The unrooted phylogeny tree was developed using the neighbor-joining method in the software MEGA 6.

Phenotypic measurements. Due to a unique linear relationship between Soil-Plant Analysis Development (SPAD) values and leaf nitrogen concentration level, a SPAD chlorophyll meter was used as a quick and nondestructive tool for estimating relative chlorophyll content of leaf ${ }^{56}$. SPAD values were taken from the fully extended flag leaf on the main stem around the heading stage using a SPAD meter (SPAD-502, Konica-Minolta, Japan). Ten readings taken from the side of the midrib of flag leaf blade were averaged, indicating the SPAD value for each plant. At least 8 plant individuals from each line were subjected to the investigation for SPAD value. Photosynthetic rate of flag leaves at 7 day after heading was measured using a portable photosynthesis system (LI-6400, LI-COR, Lincoln, USA) as described previously ${ }^{57}$. Photosynthesis rate measurement of at least 10 individuals from each line was conducted on clear days between $9 \mathrm{am}$ and 11:30 am. A digital plant stem strength detector (YYD-1A, TOP Instrument) was used to measure mechanical strength of the stems 30 days after heading, following the method as described previously ${ }^{58}$.

Determination of pigment and total $\mathbf{N}$ of flag leaves. Fresh leaves were sampled to extract chlorophyll using the method described by Lichtenthaler ${ }^{59}$. Briefly, the fresh leaves whose main veins removed were cut into small pieces weighing approximately $50 \mathrm{mg}$, were homogenized, then were added into $8 \mathrm{ml} 95 \%(\mathrm{v} / \mathrm{v})$ alcohol. Pigments were extracted at $4{ }^{\circ} \mathrm{C}$ in dark. The measurements of chlorophyll $a$, chlorophyll $b$, total absolute chlorophyll, and carotinoid concentration were conducted with Spectrophotometers (DU640, Beckman Coulter) at $665 \mathrm{~nm}, 649 \mathrm{~nm}$ and $470 \mathrm{~nm}$ with $95 \%$ ethanol as blank control, respectively. 
Leaf nitrogen content was determined in the method as described previously ${ }^{60}$. Leaf samples were inactivated at $105^{\circ} \mathrm{C}$ for $30 \mathrm{~min}$, then were dried at $80^{\circ} \mathrm{C}$ until constant weight, and were ground to fine powders. The $0.2 \mathrm{~g}$ of dry powder was digested with $5 \mathrm{ml} 98 \% \mathrm{H}_{2} \mathrm{SO}_{4}(\mathrm{w} / \mathrm{w})$ and $5 \mathrm{ml} 30 \% \mathrm{H}_{2} \mathrm{O}_{2}(\mathrm{w} / \mathrm{w})$ in a conventional high temperature digester (AMS-Westoco, France). The digested sample was diluted with distilled water to $100 \mathrm{ml}$. The nitrogen concentration in the solution was determined on an automated discrete analyzer (SmartChem 200, France). All measurements were repeated three times.

Measurement of starch, cellulose, and hemicellulose content. Flag leaves of main stem were collected, inactivated, ground through a 40 mesh sieve and stored in a dry container until use. Various cell wall components such as soluble sugar, lipid, starch, pectin, hemicellulose and cellulose were fractionated following the procedure described previously with minor modifications ${ }^{61}$. After sequentially extracted soluble sugar with potassium phosphate buffer ( $\mathrm{pH} 7.0$ ) and removed lipids with chloroform-methanol (1:1, v/v), starch were extracted using DMSO (dimethyl sulfoxide)-water $(9: 1, \mathrm{v} / \mathrm{v})$. The remaining crude cell walls were treated with $0.5 \%(\mathrm{w} / \mathrm{v})$ ammonium oxalate for pectin. The deposit was then suspended in $4 \mathrm{M} \mathrm{KOH}$ containing $1.0 \mathrm{mg} \mathrm{ml}^{-1}$ sodium borohydride, and the supernatants were neutralized and lyophilized as $\mathrm{KOH}$-extractable hemicelluloses. The remaining residues were hydrolyzed trifluoroacetic acid (TFA), washed with distilled water and the supernatants were combined as the non-KOH-extractable hemicelluloses. The remaining pellets were defined as crude cellulose.

For soluble sugar determination, total hexoses and pentoses were measured in the potassium phosphate buffer fraction. For starch assay, it was estimated as total hexoses in the DMSO fraction by anthrone $/ \mathrm{H}_{2} \mathrm{SO}_{4}$ assay ${ }^{62}$. For cellulose, the crude celluose fraction was dissolved in $67 \% \mathrm{H}_{2} \mathrm{SO}_{4}$, and the supernatants were collected for determination of hexoses as cellulose ${ }^{62}$. For hemicelluloses assay, total hexoses and pentoses were measured in the hemicellulose fraction. The pentoses were detected by orcinol/ $\mathrm{HCl}$ method ${ }^{63}$. The hexose and pentose measurements were performed using an UV/VIS Spectrometer (MAPADA, Shanghai). The experiments were performed three times.

Monosaccharide and GICA analysis of crude cell walls by GC-MS. Monosaccharide and GlcA analysis of crude cell walls was followed the method with minor modifications ${ }^{64}$. Briefly, after pectin removal as described above, the pellets were suspended in $2.5 \mathrm{ml}$ desalted solution, and hydrolyzed with TFA (2 M) at $120^{\circ} \mathrm{C}$ for $1 \mathrm{~h}$. The supernatant was dried in vacuum, then was silanized, and diluted in $1.3 \mathrm{ml}$ hexane for GC-MS analysis. GC/MS analysis was conducted with SHIMADZU GCMSQP 2010 Plus. D-glucuronic acid, D-xylose, $\mathrm{D}$-arabinose, and D-glucose were used as GlcA or monosaccharide standards. Peaks were identified by mass profiles and/or retention times of standards.

Hydroponic experiments. The 15-day-old seedlings were transferred to a standard nitrogen-deficiency rice culture solution described previously ${ }^{65}$, which was supplemented with $0.36 \mathrm{mM}, 1.44 \mathrm{mM}$, and $2.88 \mathrm{mM} \mathrm{NH}_{4} \mathrm{NO}_{3}$ representing low-N, normal-N, and high-N conditions, respectively, and were grown for 3 weeks. Hydroponics experiments were carried out in a net room with 14-h light/10-h dark conditions with an average temperature of $26^{\circ} \mathrm{C}$. Plants were grown in a container with 50L nutrient solution for each treatment of low-N, normal-N, and high-N with two biological replicates. The nutrient solution was refreshed every 3 days. After 3-week growth, the plants were measured for SPAD-value, seedling length, and biomass.

Observation of the cross-section of flag leaves. Central sections of flag leaf blades were sampled, fixed in $70 \%$ FAA, dehydrated through a series of ethanol gradient, and then embedded in paraffin wax. Leaves were cut using a rotary microtome (RM2235, Leica), and placed on lysine-treated slides, and dried for 2 days at $37^{\circ} \mathrm{C}$, de-waxed with xylene and hydrated through an ethanol gradient, stained with $0.05 \%$ toluidine blue, dehydrated with gradient alcohol and xylene dehydration, and then mounted as described previously ${ }^{66}$.

Vascular bundles of leaf mesophyll anatomy were observed under a fluorescence microscope (DM2000, Leica). The thickness of large and small vascular bundles and the distance between two consecutive small vascular bundles were measured and analyzed at 10 -fold objective magnification with digital image processing software (ImagePro Plus v. 5.1, Mitani).

For the observation under transmission electron microscope, leaves at heading stage were cut into small pieces (1 $\mathrm{mm}^{2}$ ), fixed in $2.5 \%$ glutaraldehyde in a phosphate buffer ( $\mathrm{pH} 7.2$ ), vacuum infiltrated, rinsed, and incubated overnight at $4^{\circ} \mathrm{C}$ in a solution of $1 \% \mathrm{OsO} 4$. Samples were dehydrated in a series of ethanol, infiltrated in epoxy resin at $37^{\circ} \mathrm{C}$ for $8-12 \mathrm{~h}$, and embedded in Epon 812 resin at $60^{\circ} \mathrm{C}$ for $48 \mathrm{~h}$. A series of $80 \mathrm{~nm}$ sections were cut using an ultramicrotome (EM UC7, Germany), and observed in a transmission electron microscope (Tecnai G 20 TWIN, FEI, USA).

\section{Data availability}

All data generated or analysed during this study are included in this published article and its Supplementary Information files.

Received: 1 August 2019; Accepted: 10 February 2020;

Published online: 28 February 2020

\section{References}

1. Douzery, E. J., Snell, E. A., Bapteste, E., Delsuc, F. \& Philippe, H. The timing of eukaryotic evolution: Does a relaxed molecular clock reconcile proteins and fossils? Proc. Natl Acad. Sci. USA 101, 15386-15391, https://doi.org/10.1073/pnas.0403984101 (2004).

2. Tanaka, A. \& Tanaka, R. Chlorophyll metabolism. Curr. Opin. Plant. Biol. 9, 248-255, https://doi.org/10.1016/j.pbi.2006.03.011 (2006).

3. Jiang, S. K. et al. Identification and fine mapping of $q C T H 4$, a quantitative trait loci controlling the chlorophyll content from tillering to heading in rice (Oryza sativa L.). J. Hered. 103, 720-726, https://doi.org/10.1093/jhered/ess041 (2012).

4. Juneja, A., Ceballos, R. M. \& Murthy, G. S. Effects of environmental factors and nutrient availability on the biochemical composition of algae for biofuels production: a review. Energ. 6, 4607-4638, https://doi.org/10.3390/en6094607 (2013). 
5. Peng, S. B., Laza, R. C., Garcia, F. C. \& Cassman, K. G. Chlorophyll meter estimates leaf area-based N concentration of rice. Commun. Soil. Sci. Plan. 26, 927-935, https://doi.org/10.1080/00103629509369344 (1995).

6. Yoder, B. J. \& Pettigrew-Crosby, R. E. Predicting nitrogen and chlorophyll content and concentrations from reflectance spectra (400-2500 nm) at leaf and canopy scales. Remote. Sens. Env. 53, 199-211 (1995).

7. Kumagai, E., Araki, T. \& Kubota, F. Correlation of chlorophyll meter readings with gas exchange and chlorophyll fluorescence in flag leaves of rice (Oryza sativa L.) plants. Plant. Prod. Sci. 12, 50-53, https://doi.org/10.1626/pps.12.50 (2009).

8. Goh, C. H. et al. Mitochondrial activity in illuminated leaves of chlorophyll-deficient mutant rice (OsCHLH) seedlings. Plant. Biotechnol. Rep. 4, 281-291, https://doi.org/10.1007/s11816-010-0146-z (2010).

9. Zhang, H. et al. A point mutation of magnesium chelatase OsCHLI gene dampens the interaction between CHLI and CHLD subunits in rice. Plant. Mol. Biol. Rep. 33, 1975-1987, https://doi.org/10.1007/s11105-015-0889-3 (2015).

10. Kong, W. Y. et al. The catalytic subunit of magnesium-protoporphyrin IX monomethyl ester cyclase forms a chloroplast complex to regulate chlorophyll biosynthesis in rice. Plant. Mol. Biol. 92, 177-191, https://doi.org/10.1007/s11103-016-0513-4 (2016).

11. Wu, Z. M. et al. A chlorophyll-deficient rice mutant with impaired chlorophyllide esterification in chlorophyll biosynthesis. Plant. Physiol. 145, 29-40, https://doi.org/10.1104/pp.107.100321 (2007).

12. Jiang, H. W. et al. Overexpression of SGR results in oxidative stress and lesion-mimic cell death in rice seedlings. J. Integr. Plant. Biol. 5, 375-387, https://doi.org/10.1111/j.1744-7909.2011.01037.x (2011)

13. Wang, X. C. et al. Functional characterization of the glycoside hydrolase encoding gene OsBE1 during chloroplast development in Oryza sativa. Acta Agron. Sin. 40, 2090-2097 (2014).

14. Niittylä, T. et al. A previously unknown maltose transporter essential for starch degradation in leaves. Sci. 303, 87-89, https://doi. org/10.1126/science.1091811 (2004).

15. Zhang, F. et al. Identification of a peroxisomal-targeted aldolase involved in chlorophyll biosynthesis and sugar metabolism in rice. Plant. Sci. 250, 205-215, https://doi.org/10.1016/j.plantsci.2016.06.017 (2016).

16. Lee, S. K. et al. Analysis of a triose phosphate/phosphate translocator-deficient mutant reveals a limited capacity for starch synthesis in rice leaves. Mol. Plant. 7, 1705-1708, https://doi.org/10.1093/mp/ssu082 (2014).

17. Mortimer, J. C. et al. Absence of branches from xylan in Arabidopsis gux mutants reveals potential for simplification of lignocellulosic biomass. Proc. Natl Acad. Sci. USA 107, 17409-17414, https://doi.org/10.1073/pnas.1005456107 (2010).

18. Rennie, E. A. et al. Identification of a sphingolipid $\alpha$-glucuronosyltransferase that is essential for pollen function in Arabidopsis. Plant. Cell 26, 3314-3325, https://doi.org/10.1105/tpc.114.129171 (2014).

19. Bromley, J. R. et al. GUX1 and GUX2 glucuronyltransferases decorate distinct domains of glucuronoxylan with different substitution patterns. Plant. J. 74, 423-434, https://doi.org/10.1111/tpj.12135 (2013).

20. Mortimer, J. C. et al. An unusual xylan in Arabidopsis primary cell walls is synthesised by GUX3, IRX9L, IRX10L and IRX14. Plant. J. 83, 413-426, https://doi.org/10.1111/tpj.12898 (2015).

21. Lee, C. H., Teng, Q., Zhong, R. Q. \& Ye, Z. H. Arabidopsis GUX proteins are glucuronyltransferases responsible for the addition of glucuronic acid side chains onto xylan. Plant. Cell Physiol. 53, 1204-1216, https://doi.org/10.1093/pcp/pcs064 (2012).

22. Yu, H. H., Xie, W. B., Li, J., Zhou, F. S. \& Zhang, Q. F. A whole-genome SNP array (RICE6K) for genomic breeding in rice. Plant. Biotechnol. J. 12, 28-37, https://doi.org/10.1111/pbi.12113 (2013).

23. Sterling, J. D. et al. Functional identification of an Arabidopsis pectin biosynthetic homogalacturonan galacturonosyltransferase. Proc. Natl Acad. Sci. USA 103, 5236-5241, https://doi.org/10.1073/pnas.0600120103 (2006).

24. Oikawa, A. et al. An integrative approach to the identification of Arabidopsis and Rice genes involved in xylan and secondary wall development. PLoS One 5, e15481 (2010).

25. Peng, S. B., Garcia, F. V., Laza, R. C. \& Cassman, K. G. Adjustment for specific leaf weight improves chlorophyllmeter's estimate of rice leaf nitrogen concentration. Agron. J. 85, 987-990, https://doi.org/10.2134/agronj1993.00021962008500050005x (1993).

26. Wang, L. Q. et al. Expression profiling and integrative analysis of the CESA/CSL superfamily in rice. BMC Plant. Biol. 10, 282, https:// doi.org/10.1186/1471-2229-10-282 (2010).

27. Chiniquy, D. et al. Three novel rice genes closely related to the Arabidopsis IRX9, IRX9L, and IRX14 genes and their roles in xylan biosynthesis. Front. Plant. Sci. 4, 1-13, https://doi.org/10.3389/fpls.2013.00083 (2013).

28. Verbančič, J., Lunn, J. E., Stitt, M. \& Persson, S. Carbon supply and the regulation of cell wall synthesis. Mol. Plant. 11, 75-94, https:// doi.org/10.1016/j.molp.2017.10.004 (2018).

29. Li, C. L. et al. A rice plastidial nucleotide sugar epimerase is involved in galactolipid biosynthesis and improves photosynthetic efficiency. PLoS Genet. 7, e1002196 (2011).

30. Atlassi, P. V., Nabipour, M. \& Meskarbashee, M. Effect of salt stress on chlorophyll content, fluorescence, $\mathrm{Na}^{+}$and $\mathrm{K}^{+}$ions content in rape plants (Brassica napus L.). Asian. Agric. Res. 3, 28-37 (2009).

31. Hörtensteiner, S. Chlorophyll degradation during senescence. Annu. Rev. Plant. Biol. 57, 55-77, https://doi.org/10.1146/annurev. arplant.57.032905.105212 (2006).

32. Loh, F. C. W., Grabosky, J. C. \& Bassuk, N. L. Using the SPAD 502 meter to assess chlorophyll and nitrogen content of benjamin fig and cottonwood leaves. HortTechnology 12, 682-686, https://doi.org/10.21273/HORTTECH.12.4.682 (2002).

33. Ajia, L. T. et al. QTL analysis for chlorophyll content in four grain-filling stage in rice. Acta Agron. Sin. 34, 61-66 (2008),

34. Sun, X. X. et al. Analysis on quantitative trait loci associated with leaf chlorophyll content and their interactions with environment at late growth stage of rice. J. Appl. Ecol. 19, 2651-2655 (2008).

35. Huang, L. C. et al. Genetic dissection for chlorophyll content of the top three leaves during grain filling in rice (Oryza sativa L.). J. Plant. Growth Regul. 34, 381-391, https://doi.org/10.1007/s00344-014-9474-5 (2015).

36. Hu, S. P. et al. Correlation and quantitative trait loci analyses of total chlorophyll content and photosynthetic rate of rice (Oryza sativa) under water stress and well-watered conditions. J. Integr. Plant. Biol. 51, 879-888, https://doi.org/10.1111/j.1744-7909.2009.00846.x (2009).

37. Lei, D. Y., Tan, L. B., Liu, F. X., Chen, L. Y. \& Sun, C. Q. Identification of heat-sensitive QTL derived from common wild rice (Oryza rufipogon Griff.). Plant. Sci. 201-202, 121-127, https://doi.org/10.1016/j.plantsci.2012.12.001 (2013).

38. Jiang, G. H., He, Y. Q., Xu, C. G., Li, X. H. \& Zhang, Q. F. The genetic basis of stay-green in rice analyzed in a population of doubled haploid lines derived from an indica by japonica cross. Theor. Appl. Genet. 108, 688-698, https://doi.org/10.1007/s00122-003-1465-z (2004).

39. Liu, X. et al. Genetic dissection of leaf-related traits using 156 chromosomal segment substitution lines. J. Plant. Biol. 58, 402-410, https://doi.org/10.1007/s12374-015-0402-4 (2015).

40. Lim, J. H. \& Paek, N. C. Quantitative trait locus mapping and candidate gene analysis for functional stay-green trait in rice. Plant. Breed. Biotech. 3, 95-107, https://doi.org/10.9787/PBB.2015.3.2.095 (2015).

41. Rennie, E. A. et al. Three members of the Arabidopsis glycosyltransferase family 8 are xylan glucuronosyltransferases. Plant. Physiol. 159, 1408-1417, https://doi.org/10.1104/pp.112.200964 (2012).

42. Ali, M. L., Sanchez, P. L., Yu, S. B., Lorieux, M. \& Eizenga, G. C. Chromosome segment substitution lines: a powerful tool for the introgression of valuable genes from Oryza wild species into cultivated rice (O. sativa). Rice 3, 218-234, https://doi.org/10.1007/ s12284-010-9058-3 (2010).

43. Paran, I. \& Zamir, D. Quantitative traits in plants: beyond the QTL. Trends Genet. 19, 303-306, https://doi.org/10.1016/s01689525(03)00117-3 (2003).

44. McCouch, S. R. CGSNL (committee on gene symbolization, nomenclature, linkage, rice genetics cooperative). Gene nomenclature system for rice. Rice 1, 72-84, https://doi.org/10.1007/s12284-008-9004-9 (2008). 
45. Murray, M. G. \& Thompson, W. F. Rapid isolation of high molecular weight plant DNA. Nucleic Acids Res. 8, 4321-4325, https://doi. org/10.1093/nar/8.19.4321 (1980).

46. Wang, S. C., Basten, C. J. \& Zeng, Z. B. Windows QTL Cartographer version 2.5. http://statgen.ncsu.edu/qtlcart/WQTLCart.htm, (Department of Statistics, North Carolina State University, 2007).

47. Churchill, G. A. \& Doerge, R. W. Empirical threshold values for quantitative trait mapping. Genet. 138, 963-971 (1994).

48. Vandesompele, J. et al. Accurate normalization of real-time quantitative RT-PCR data by geometric averaging of multiple internal control genes. Genome Biol. 3, research0034.1-0034.11, https://doi.org/10.1186/gb-2002-3-7 (2002).

49. Livak, K. J. \& Schmittgen, T. D. Analysis of relative gene expression data using realtime quantitative PCR and the $2^{-\Delta \Delta(\mathrm{CT})}$ method. Methods 25, 402-408, https://doi.org/10.1006/meth.2001.1262 (2001).

50. Yuan, B., Shen, X. L., Li, X. H., Xu, C. G. \& Wang, S. P. Mitogen-activated protein kinase OsMPK6 negatively regulates rice disease resistance to bacterial pathogens. Planta 226, 953-960, https://doi.org/10.1007/s00425-007-0541-z (2007).

51. Lin, Y. J. \& Zhang, Q. Optimising the tissue culture conditions for high efficiency transformation of indica rice. Plant. Cell Rep. 23, 540-547, https://doi.org/10.1007/s00299-004-0843-6 (2005).

52. Cai, Y. et al. Multiple cytosolic and transmembrane determinants are required for the trafficking of SCAMP1 via an ER-Golgi-TGNPM pathway. Plant. J. 65, 882-896, https://doi.org/10.1111/j.1365-313X.2010.04469.x (2011).

53. Zhang, Y. et al. A highly efficient rice green tissue protoplast system for transient gene expression and studying light/chloroplastrelated processes. Plant. Methods 7, 30-43, https://doi.org/10.1186/1746-4811-7-30 (2011).

54. Goodstein, D. M. et al. Phytozome: a comparative platform for green plant genomics. Nucleic Acids Res. 40, 1178-1186, https://doi. org/10.1093/nar/gkr944 (2012).

55. Darabi, M. \& Seddigh, S. Computational study of biochemical properties of Ribulose-1, 5-Bisphosphate Carboxylase/Oxygenase (RuBisCO) enzyme in C p $_{3}$ plants. J. Plant. Biol. 60, 35-47, https://doi.org/10.1007/s12374-016-0211-4 (2017).

56. Xiong, D. L. et al. SPAD-based leaf nitrogen estimation is impacted by environmental factors and crop leaf characteristics. Sci. Rep. 5, 13389, https://doi.org/10.1038/srep13389 (2015).

57. Wang, Q. X. et al. Genetic architecture of natural variation in rice chlorophyll content revealed by a genome-wide association study. Mol. Plant. 8, 946-957, https://doi.org/10.1016/j.molp.2015.02.014 (2015).

58. Wu, X. R. et al. Characteristics of lodging resistance of super-hybrid indica rice and its response to nitrogen. Sci. Agric. Sin. 48, 2705-2717 (2015).

59. Lichtenthaler, H. K. Chlorophylls and carotenoids: pigments of photosynthetic biomembranes in Methods in Enzymology (eds, Colowick, S. P. \& Kaplan, N. O.) 350-382 (San Diego: Academic, 1987).

60. Yang, M. et al. Genome-wide association studies reveal the genetic basis of ionomic variation in rice. Plant. Cell 30, 2720-2740, https://doi.org/10.1105/tpc.18.00375 (2018).

61. Li, A. et al. An integrative analysis of four CESA isoforms specific for fiber cellulose production between Gossypium hirsutum and Gossypium barbadense. Planta 237, 1585-1597, https://doi.org/10.1007/s00425-013-1868-2 (2013).

62. Fry, S. C. The growing plant cell wall: chemical and metabolic analysis (Harlow: Longman, 1988).

63. Dische, Z. Color reactions of carbohydrates in Methods Carbohydrate Chemistry (eds, Whistler, R. L. \& Wolfrom, M. L.) 477-512 (New York: Academic, 1962).

64. Wang, Y. T. et al. Ammonium oxalate-extractable uronic acids positively affect biomass enzymatic digestibility by reducing lignocellulose crystallinity in Miscanthus. Bioresour. Technol. 196, 391-398, https://doi.org/10.1016/j.biortech.2015.07.099 (2015).

65. Yoshida, S., Forno, D. A., Cock, J. H. \& Gomez, K. A. Laboratory manual for physiological studies of rice (ed. 2nd) 57-63 (Manila: International Rice Research Institute, 1972).

66. Cheng, K., Du, H. \& Ouyang, Y. D. Paraffin section of rice tissue. Bio 101, e1010140, https://doi.org/10.21769/BioProtoc.1010140 (2018).

\section{Acknowledgements}

This work was supported by funding from the National Natural Science Foundation of China (no. 31671656), the Ministry of Agriculture of China (2016ZX08009002), the Fundamental Research Funds for the Central Universities (2662018YJ025), and the National High Technology Research and Development of China (no. 2014AA10A604). We thank Professor Ping Liu for assistance in language editing.

\section{Author contributions}

S.Y. conceived and designed the research; D.G., W.S. and D.W. performed the experiments. D.G. and H.D. developed the mapping populations, R.Z. conducted the analysis of cellular component. S.Y. and D.G. analyzed data and wrote the manuscript.

\section{Competing interests}

The authors declare no competing interests.

\section{Additional information}

Supplementary information is available for this paper at https://doi.org/10.1038/s41598-020-60593-3.

Correspondence and requests for materials should be addressed to S.Y.

Reprints and permissions information is available at www.nature.com/reprints.

Publisher's note Springer Nature remains neutral with regard to jurisdictional claims in published maps and institutional affiliations.

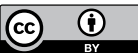

Open Access This article is licensed under a Creative Commons Attribution 4.0 International License, which permits use, sharing, adaptation, distribution and reproduction in any medium or format, as long as you give appropriate credit to the original author(s) and the source, provide a link to the Creative Commons license, and indicate if changes were made. The images or other third party material in this article are included in the article's Creative Commons license, unless indicated otherwise in a credit line to the material. If material is not included in the article's Creative Commons license and your intended use is not permitted by statutory regulation or exceeds the permitted use, you will need to obtain permission directly from the copyright holder. To view a copy of this license, visit http://creativecommons.org/licenses/by/4.0/.

(c) The Author(s) 2020 